\title{
An Ultraluminous IRAS Galaxy with an Extremely Soft X-Ray Spectrum ${ }^{1}$
}

\author{
X. Y. Xia, ${ }^{2}$ Th. Boller, ${ }^{3}$ and Z. G. Deng ${ }^{4}$ \\ ${ }^{2}$ Department of Physics, Tianjin Normal University, Tianjin 300074, \\ China \\ ${ }^{3}$ Max-Planck-Institute für Extraterrestrische Physik \\ Karl-Schwarzschild-Strasse 1, 85740 Garching bei München, Germany \\ ${ }^{4}$ Department of Physics, Graduate School, Chinese Academy of Science, \\ 100039 Beijing, China
}

Redshift surveys of the extragalactic IRAS sources have revealed a class of galaxies, ultraluminous infrared galaxies (ULIRGs), that radiate an enormous amount of energy in the far-infrared (Soifer et al. 1986), typically $L_{\mathrm{IR}} \geq 10^{12} \mathrm{~L}_{\odot}$, which is comparable to the luminosities of quasars. The energy source for most ULIRGs is believed to be massive nuclear starbursts triggered by galaxy interactions or mergers (Rigopoulou et al. 1996, Crawford et al. 1996). There is much debate about the evolution of ULIRGs. Sanders et al. (1988) suggested that ULIRGs are forming QSOs. However, it is not clear how merging galaxies evolve to QSOs. Meanwhile, observations and N-body simulations show that mergers between disk galaxies can form ellipticals. Some ULIRGs, such as Arp 220 and NGC 6240, do have elliptical characteristics. What is the evolutionary connection between QSOs and elliptical galaxies? About $10 \%$ of ULIRGs are IR QSOs or Seyfert 1 galaxies, on the basis of their optical spectra and appearance. Some of them are hosted in ellipticals (Hutchings et al. 1988, Lipari et al. 1994). Also, most of them are strong Fe II emitters. Studying this subsample of ULIRGs in detail may give some clues to understanding the evolutionary process mentioned above.

We have performed a cross identification of 131 ULIRGs compiled from the QDOT IRAS redshift survey with the ROSAT all-sky survey (RASS). Among the identified sources is IRAS $10026+4347$, which is a Seyfert 1 galaxy or IR QSO according to its optical image, luminosity, and spectrum. Its optical spectrum shows that it is a strong Fe II emitter. Figure 1 shows the infrared color-color diagram. We can see from Fig. 1 that IRAS $10026+4347$ is located above the power-law line. For this source, $394 \mathrm{X}$-ray photons have been detected by the RASS during a total the exposure time of 508 seconds. The redshift of IRAS $10026+4347$ is $z=0.178$, so its soft X-ray luminosity is $2 \times 10^{45} \mathrm{ergs}^{-1}$. The analysis of ROSAT all-sky survey data shows that it is a point-like source, although it is possible that there exists a soft X-ray halo around it. However the spectrum is extremely soft; the RASS data show that IRAS 10026+4347 emits more than $70 \%$ of its flux below $0.5 \mathrm{keV}$ and there are no photons above $1.1 \mathrm{keV}$, as shown in Fig. 2. 'Supersoft' AGNs have been found in the ROSAT

\footnotetext{
${ }^{1}$ This work is supported by the National Natural Science Foundation of China.
} 


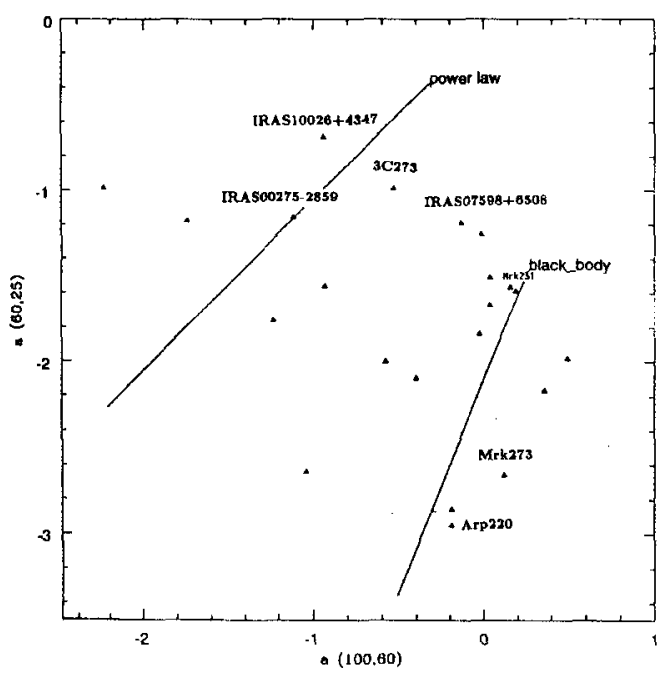

Figure 1. The infrared color-color diagram for 22 ULIRGs identified with ROSAT RASS and pointed-observation sources. IRAS $10026+4347$ is above the power-law line.

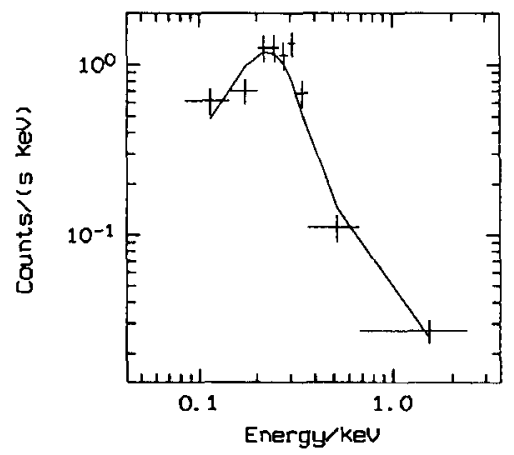

Figure 2. The soft X-ray spectrum of IRAS $10026+4347$ 
pointed catalog (Singh et al. 1995), but these AGNs are not as bright in the soft X-ray band as IRAS 10026+4347. From its optical and infrared properties and soft X-ray luminosity, IRAS $10026+4347$ is more like normal QSOs than IR QSOs such as IRAS 00275-2859 and IRAS 07598+6508. However its soft X-ray spectrum is extremely soft, and therefore, this ultraluminous IR QSO is a very special and interesting object to be studied.

\section{References}

Crawford, T., et al. 1996, in press.

Hutchings, J. B., et al. 1988, AJ, 95, 1575.

Lipari, S., et al. 1994, ApJ, 427, 174.

Rigopoulou, D., et al. 1996, MNRAS, 278, 1049.

Sanders, D. B., et al. 1988, ApJ, 325, 74.

Singh, K.P., et al. 1995, ApJ, 455, 456.

Soifer, B. T., et al. 1986, ApJ, 303, L41.

Xia, X. Y., et al., in preparation. 